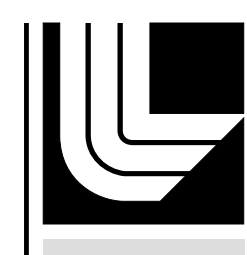

Understanding High Voltage Vacuum Insulators for Microsecond Pulses

Javedani, J.B., Goerz, D.A., Houck, T.L., Lauer, E.J., Speer, R.D., Tully, L.K., Vogtlin, G.E.

August 15, 2007

IEEE International Pulsed Power Conference Albuquerque, NM, United States June 17, 2007 through June 22, 2007 
This document was prepared as an account of work sponsored by an agency of the United States Government. Neither the United States Government nor the University of California nor any of their employees, makes any warranty, express or implied, or assumes any legal liability or responsibility for the accuracy, completeness, or usefulness of any information, apparatus, product, or process disclosed, or represents that its use would not infringe privately owned rights. Reference herein to any specific commercial product, process, or service by trade name, trademark, manufacturer, or otherwise, does not necessarily constitute or imply its endorsement, recommendation, or favoring by the United States Government or the University of California. The views and opinions of authors expressed herein do not necessarily state or reflect those of the United States Government or the University of California, and shall not be used for advertising or product endorsement purposes. 


\title{
Understanding High Voltage Vacuum Insulators for Microsecond Pulses *
}

\author{
J. B. Javedani $^{\xi}$, D. A. Goerz, T. L. Houck, E.J. Lauer, R. D. Speer, L. K. Tully, G.E. Vogtlin \\ Lawrence Livermore National Laboratory, L-154 \\ 7000 East Avenue, Livermore, CA, USA
}

\begin{abstract}
High voltage insulation is one of the main areas of pulsed power research and development since the surface of an insulator exposed to vacuum can fail electrically at an applied field more than an order or magnitude below the bulk dielectric strength of the insulator. This is troublesome for applications where high voltage conditioning of the insulator and electrodes is not practical and where relatively long pulses, on the order of several microseconds, are required.

Here we give a summary of our approach to modeling and simulation efforts and experimental investigations for understanding flashover mechanism. The computational work is comprised of both filed and particle-in-cell modeling with state-of-the-art commercial codes. Experiments were performed in using an available 100$\mathrm{kV}, 10-\mu \mathrm{s}$ pulse generator and vacuum chamber. The initial experiments were done with polyethylene insulator material in the shape of a truncated cone cut at $+45^{\circ}$ angle between flat electrodes with a gap of $1.0 \mathrm{~cm}$. The insulator was sized so there were no flashovers or breakdowns under nominal operating conditions. Insulator flashover or gap closure was induced by introducing a plasma source, a tuft of velvet, in proximity to the insulator or electrode.
\end{abstract}

\section{Introduction}

Current best practices in high voltage insulator design have evolved over several decades and have been based primarily on empirical results without the benefit of substantial computational studies, often limited to field modeling only [1].

It is understood that cathode-initiated surface breakdown can result from explosive emission of electrons from either 1) micro-protrusions on the electrode when average electric fields are $>100 \mathrm{kV} / \mathrm{cm}$ or 2) dielectric fibers, whiskers, lint, and debris when average electric fields are $<100 \mathrm{kV} / \mathrm{cm}$. Anode initiated breakdown is the result of the high stress that is placed at the ATJ (anode triple junction) from the applied electric potential that either; a) causes bulk breakdown due to ohmic heating, or b) caused surface breakdown due to a

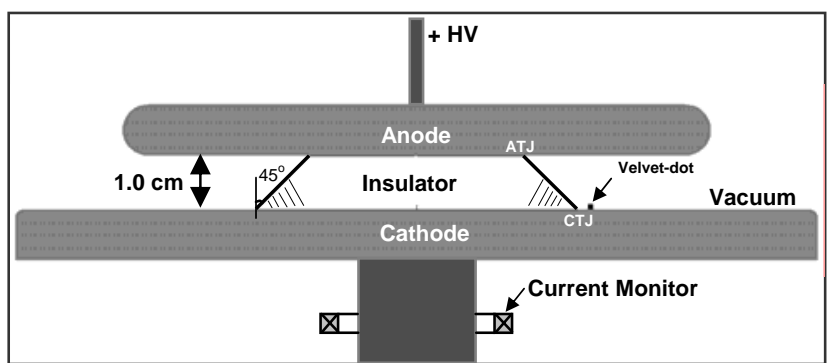

Figure 1. Setup geometry to test an insulator for surfaceflashover studies.

whisker at the ATJ.

The approach we have taken is to develop a thorough understanding of the fundamental mechanisms governing surface flashover of high voltage vacuum insulators using computational methods, coupled with experiments to validate and quantify expected behaviors. This approach centers around studying the effect of an electron-emitter, in the form of a $1.0 \mathrm{~mm}$ dia. tuft of velvet, when exposed to high field in the presence of a $+45^{\circ}$ high density polyethylene insulator [2]. See Figure 1.

\section{Modeling, Simulation and Theory}

Specialized software was required to meet the analytical and computational needs of this challenge. Two of the heavily used codes were: 1) TriComp $^{\mathrm{TM}}$ and AMaze ${ }^{\mathrm{TM}}$ Suites include 2D \& 3D conformal mesh generators, finite element electrostatic field solvers, and charged particle optics with a zoom-in option and 2) LSP ${ }^{\mathrm{TM}}$ - A 3D time dependent, electromagnetic particle-in-cell (PIC) code designed for large scale plasma simulations. LSP is an open source code and runs in parallel on multiple CPUs on Linux based computers.

\section{A. Field Modeling}

The ability to finely resolve critical features of possibly small defects in rather large problem spaces is important in studying insulator flashover mechanisms. We explored the limit on accuracy for calculating the magnitude of electric field and potential in the triple point region of the insulator/vacuum interface. We did this using the TriComp electrostatic solver with its zoom feature, and

\footnotetext{
* This work was performed under the auspices of the U. S. Department of Energy (DOE) by the University of California, Lawrence Livermore National Laboratory (LLNL) under Contract No. W-7405-Eng-48.

รemail: javedani1@llnl.gov
} 
then compared and validated the results to an analytical approach. It can be shown mathematically that the field increases as $\mathrm{r}^{v}$ approaching the ATJ and decreases as $\mathrm{r}^{v}$ approaching the CTJ (cathode triple junction) for a positive insulator angle where $r$ is the distance to the respective triple junction and $v$ is a positive number [3], [4].

\section{B. LSP Simulation Modeling}

LSP simulations were performed on a relatively small (34-cpu) Linux cluster. LSP at first was run to simulate; 1) velvet emission and 2) secondary electron emission, based on a probability yield matrix involving energy and angle. With the SEE (Secondary Electron Emission) physics added to the code, we ran, 3) Simulation of electron avalanche from CTJ with angled insulator in 3-D, representing an electron avalanche originating at the CTJ of an angled insulator. Even for a reduced geometry where the electrodes are spaced only $0.5 \mathrm{~mm}$ apart, a $1 \mathrm{~ns}$ simulation time takes 10 hours using 20 processors. Figure 2 shows results at the beginning of emission (top) and about 0.03 ns into the pulse when current is almost fully established. A side view of the simulation is shown.

A few secondaries are produced at the edge of the emission site, but these electrons follow the primary beam across the gap and do not avalanche across the surface. Future efforts will include varying the angle of the insulator from the perpendicular to determine the point where an avalanche is no longer sustained and looking at the effect of charge deposits on the insulator surface. From experiments we know that the insulator flashes over as soon as the expanding plasma touches the surface. Thus, we are looking at the physics that are not included in the simulation to understand the missing mechanism.

\section{Future Modeling and Simulation Challenges}

We have successfully demonstrated particle emission including secondary electron emission, charging of dielectrics and space charge effects on orbits in our simulations with LSP. Physics phenomena that need further exploration include desorption/ionizing of neutrals, UV induced photoemission, SEE emission from an angled insulator, and initial charge deposits on the insulator. More importantly, we are developing the ability to simulate the electron avalanche model proposed by E.J. Lauer, with the time-dependent PIC code. In his model Lauer assumes a localized, positive charge distribution in the insulator surface near the ATJ. A 3-D electrostatic code is then used to calculate the fields $E_{t}$ and $E_{n}$ from the applied voltage and the charge. Next, a Mathematica ${ }^{\mathrm{TM}}$ script is used to solve the equations for a time step and determine the new charge distribution. The 3D electrostatic code calculates the new fields, $E_{t}$ and $E_{n}$. This iterative process is repeated to step the avalanche across the insulator [5]. Eventually we would like to incorporate this procedure into a time dependent PIC code such as LSPTM.

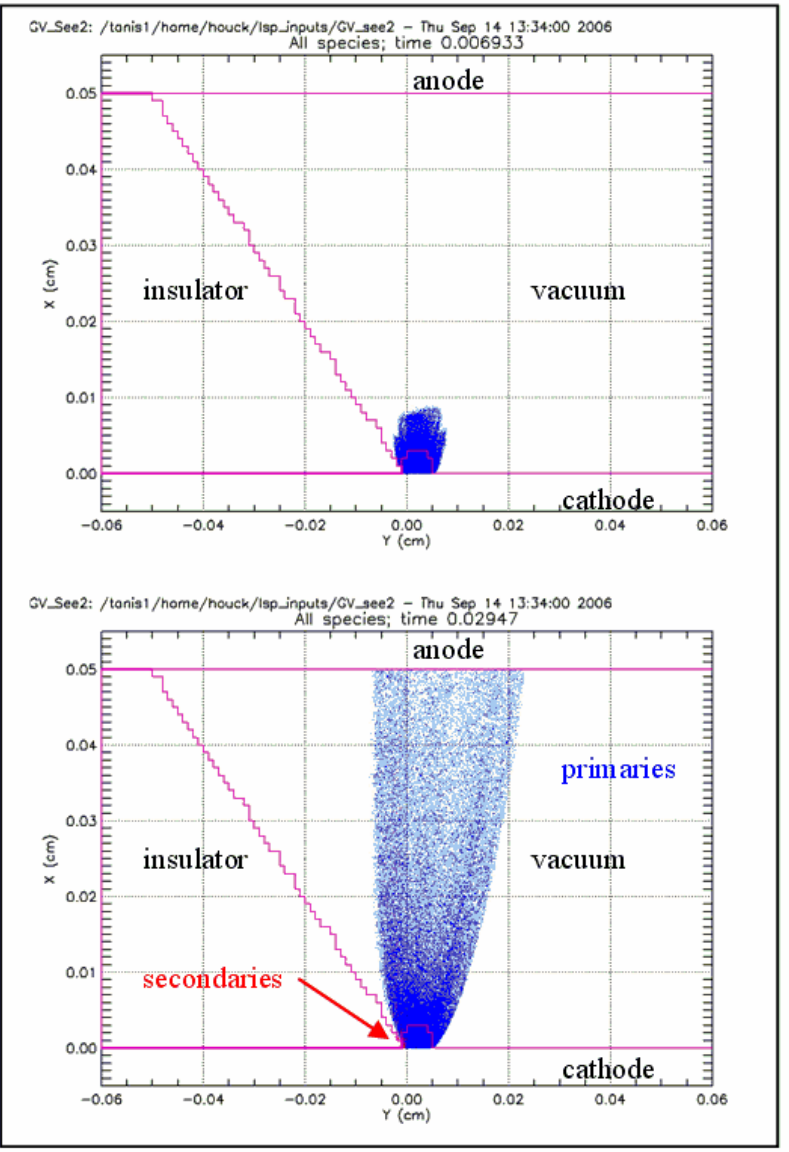

Figure 2. Emission from the CTJ: side view.

\section{Experiment}

A high voltage test stand for vacuum insulator studies was designed and constructed [2], [6]. The test stand is comprised of a vacuum chamber, high voltage pulser, and electrode fixtures for insulator testing. The vacuum of the chamber is maintained with a cryogenic pump (CTI Cryo-Torr 8 cold head) with air pumping speed of 1500 liters/s. Testing was done at pressures of less than 5 micro-torr. The vacuum chamber is shown in Figure 3.

The applied voltage was generated by a variable voltage $500 \mathrm{~J}, 100 \mathrm{nF}$ capacitive discharge unit (CDU) that incorporates a triggered spark-gap series switch, waveform shaping components, a triggered spark-gap crowbar switch, and a coaxial cable output. The CDU is capable of producing up to $100 \mathrm{kV}$ voltage pulses of 180 ns rise-time with a pulse-width up to $10 \mu$ s into a resistive load. The grounded cathode was the lower plate with a current transformer around the supporting stalk. A 250 ohm resistor in series with the CDU output was used to limit the maximum current. Normally the crowbar is activated after $5 \mu \mathrm{s}$. Spacing between the electrodes was nominally set at $1.0 \mathrm{~cm}$. The pulser is based on a design developed for the Heavy Ion Fusion Injector for LLNL's re-circulating induction accelerator [7]. 


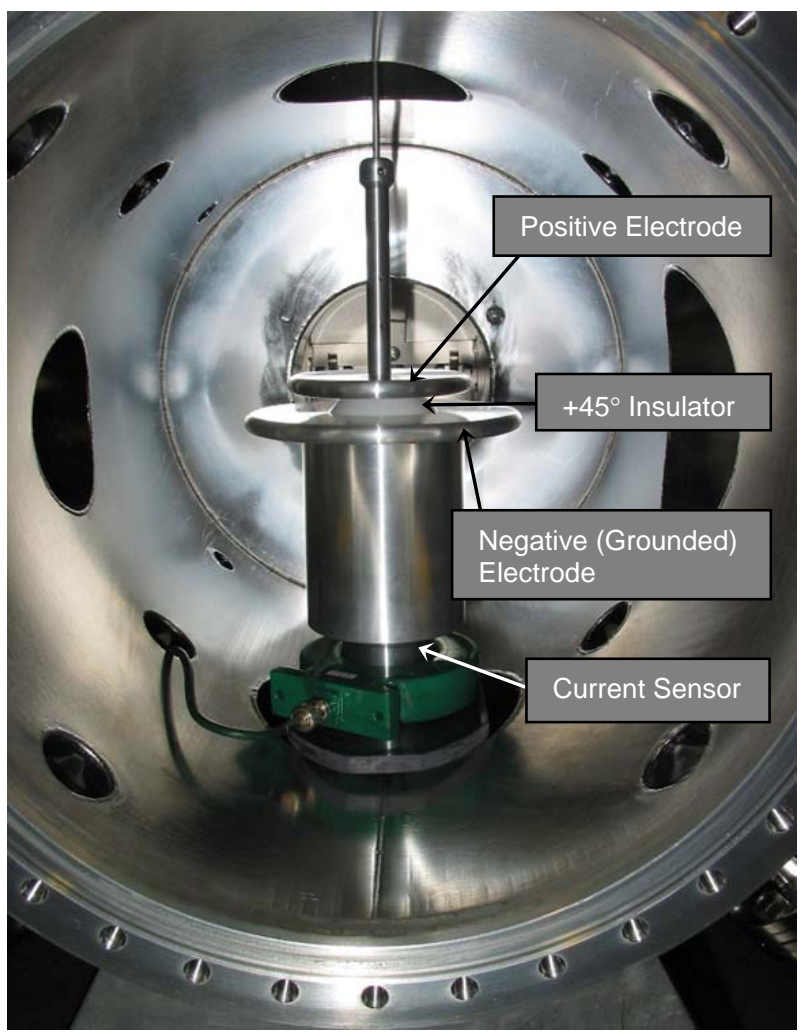

Figure 3. Test configuration of a $+45^{\circ}$ insulator.

\section{A. Electrodes, Dielectric and Surface Preparation}

Electrodes were aluminum with a 32 micro-inch finish. For some of the experiments, the cathode was anodized in accordance with MIL-A-8625C to produce a type III (hard), Class-1 (non-dyed) coating with a specified thickness of $0.002-0.004$ inches. The cathode diameter was $15.0 \mathrm{~cm}$ and the anode diameter was $10.0 \mathrm{~cm}$.

HD polyethylene was the insulator material. The overall load capacitance (with the nominal HD polyethylene insulator) can be calculated from displacement current measurements (peaks at $45 \mathrm{Amp}$, for $100 \mathrm{kV}$ CDU charge) to be about $17 \mathrm{pF}$. The insulators were also machined to a 32 micro-inch finish.

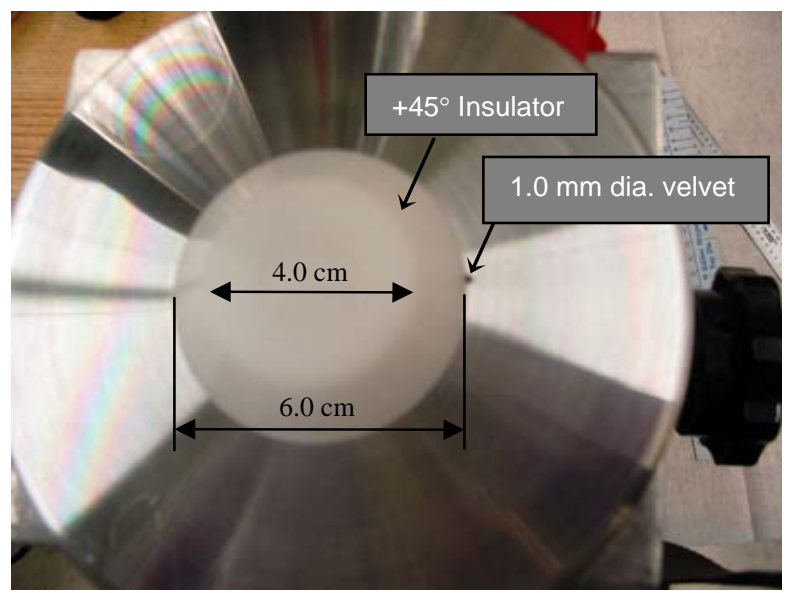

Figure 4. Top view of the insulator \& velvet.

\section{B. Velvet Tuft as the Electron Emitter}

In many of the tests, small pieces of synthetic velvet were used to facilitate flashover. Double Eagle (black in color), courtesy of NRL [8], was the velvet of choice in forming plasmas at relatively low field stresses; at 80-100 $\mathrm{kV} / \mathrm{cm}$ levels on the first shot and after conditioning at $40-60 \mathrm{kV} / \mathrm{cm}$ levels. The velvet was nominally cut with a circular base of $1 \mathrm{~mm}$ diameter, a height of about $1.5 \mathrm{~mm}$, and was comprised of about six tufts of fibers. The velvet was then attached to the surface with a small dot of double stick tape (3M, \# 465 light industrial, adhesive transfer type). Figure 4 shows a velvet-dot positioned at the CTJ of an HD polyethylene insulator.

\section{Diagnostics}

A liquid resistive voltage divider attached to the drive cable at the entrance to the vacuum chamber monitored the output of the CDU. The current was measured with a Pearson 110 current transformer installed around the cathode support stalk. Both of these monitors are relatively slow ( $20 \mathrm{MHz}$ bandwidth), although sufficient for the plasma studies. For future experiments, we plan to install D-dot probes with sub-nanosecond response in the cathode (at ground potential) under the insulator.

\section{Measurements and Observations}

Before installation of the velvet on the cathode electrode, the voltage hold off of the bare Aluminum electrodes with $1.0 \mathrm{~cm}$ gap was tested at $+100 \mathrm{kV}$ and 5 $\mu$ s long CDU pulses. There were no breakdowns in this configuration. We proceeded with the velvet-only test in order to characterize the velvet/velvet generated plasma. The insulator was then introduced to the configuration. Placement of the velvet-dot in the vicinity of the CTJ and ATJ followed. A fresh velvet-dot was installed at the beginning of each series. Normally each shot was repeated 3 times for the velvet-only and velvet-dot at the CTJ cases. The turn-around time between shots was $\sim 10$ minutes. Figure 5 shows selected voltage waveform for several velvet-CTJ positions at an applied discharge voltage of $80 \mathrm{kV}$.

For the velvet-dot at the ATJ series, the shots were repeated as many as 10 times. The test-insulators geometry was in the shape of truncated $45^{\circ}$ cones, $1.0 \mathrm{~cm}$ thick, and a base diameter of $6.0 \mathrm{~cm}$. The velvet-dot was $1.5 \mathrm{~mm}$ thick with a diameter of $1.0 \mathrm{~mm}$.

Below a summary of key measurements and observations that were made in each shot series is given: 1) Velvet Only: to characterize the plasma and ChildLangmuir current limit. 2) Velvet-dot in the Vicinity of CTJ, to characterize plasma-insulator interaction in low field of CTJ region. 3) Velvet-dot at ATJ: to characterize plasma-insulator in the high field region of ATJ.

Summary of Observations made with CTJ initiation

1. Plasma and not the UV from the plasma was the culprit in causing insulator flashover. 
2. The velvet only case shows a speed of $2.58 \mathrm{~cm} / \mu$ s (100 $\mathrm{kV}$ charge) across the gap once it is conditioned. The current is no longer Child Langmuir limited and increases steeply after the plasma arrives or is launched at the CTJ.

3. Breakdown takes place with no precursor voltage droop or current for the velvet placed at the CTJ. The reproducibility of this phenomenon was consistent. The electrons that are pulled out of the velvet glide along the surface of the insulator freely at time scales too fast to be measured by our relatively $(20 \mathrm{MHz}$ ) slow probes.

4. The plasmas produced by velvet-dots at 2.0, 4.0 and 6.0 $\mathrm{mm}$ away from CTJ show average velocities of 2.25, 1.75 , and $1.55 \mathrm{~cm} / \mu \mathrm{s}$ along the cathode - voltage waveforms are shown in Figure 5. This data indicates that plasma slowed down as it expanded.

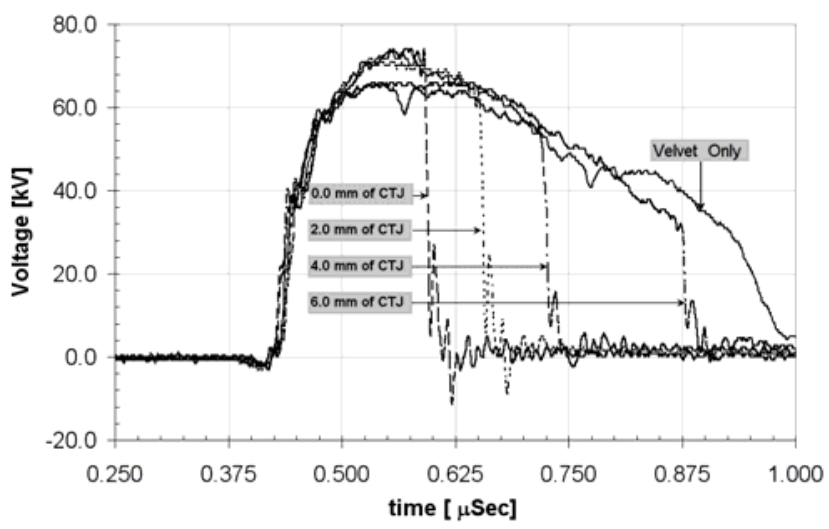

Figure 5. Voltage waveform for $+80 \mathrm{kV}$ discharge for several velvets at CTJ positions.

\section{Summary of Observations made with ATJ initiation}

1. None of the breakdowns at the ATJ can be attributed to bulk breakdown since there were no observable damages (e.g., treeing effect) to the insulator. The amount of energy transferred from the CDU to the insulator during the ATJ initiated flash over is only about 0.1 Joule. This is not enough energy deposition to cause permanent damage.

2. The anode initiated breakdowns were all surface breakdowns induced by the presence of the velvet. To our knowledge no one has reported on this mechanism for breakdown. It is not intuitively obvious why the electrons initiated at the ATJ would not just get absorbed in the neighboring anode and would instead avalanche on the surface of the insulator making it to the cathode.

3. Breakdowns on the first few shots of a newly installed velvet occurred with a time delay (less than $1 \mu \mathrm{s}$ ). The breakdowns would happen on the voltage rise after these preliminary shots.

4. The voltage collapse is too fast to be resolved by either of the voltage or current probes in any of these discharges. Faster response probes are needed to collect accurate data.

\section{Summary}

Modeling and simulation efforts included applications and extension of many configuration tools including TriComp ${ }^{\mathrm{TM}}$, $\mathrm{AMaze}^{\mathrm{TM}}$, and $\mathrm{LSP}^{\mathrm{TM}}$ codes. Field modeling examined and quantified the resolution possible for simulation field enhancements and possible defect mechanisms. Particle-in-cell modeling was used to simulate and study the plasma physics at play in the mechanisms leading to onset of insulator flashover.

We assembled a high voltage test stand for vacuum insulator studies with the minimal set of diagnostics to perform our initial experiments. Velvet was used to represent possible defect materials and be a plasma source for experimentation at relatively low field stress levels with +45 degree HD Polyethylene as the insulator. Our measurements and observations indicated that surface flashover starting near the cathode triple junction is triggered by explosive electron emission from a nearby micro-protrusion or dielectric whisker/debris. Flashovers near the anode triple junction did not produce residual tree-like damage patterns, due to insufficient energy in the discharge.

Our theoretical studies led to a description and supporting analysis of a possible mechanism for anode initiated surface flashover that involves an electron avalanche process starting near the anode, not a mechanism involving bulk dielectric breakdown.

The events leading to a flashover are relatively long ( 1.0E-06 seconds) but the flashover of the insulator itself happens at much faster times ( 1.0E-09 seconds). Faster diagnostics are being fabricated and purchased to resolve the flashover time. The data taken by the fast probes will be used to further benchmark the PIC code.

\section{REFERENCES}

1) W.A. Stygar, et al., "Improved Design of a HighVoltage Vacuum Interface, Phys. Rev. ST Accel. Beams 8050401 (2005).

2) J.B. Javedani, et. al., "Understanding and Improving High Voltage Vacuum Insulators for Microsecond Pulses," March, 2007, UCRL-TR-228713.

3) M.S. Chung, T.S. Choi, B.G. Yoon, "Theoretical analysis of the field enhancement in a two-dimensional triple junction”, Applied Surface Science, 2005.

4) L.K. Tully, et. al., "Electrostatic Modeling of Vacuum Insulator Triple Junctions," this proceeding.

5) E. J. Lauer, "Electron Avalanche Model of Dielectric Vacuum Surface Breakdown,” for submission to Journal of Applied Physics for publication.

6) T.L. Houck, et. al., "Study of Vacuum Insulator Flashover for Pulse Lengths of Multi-Microseconds," Linear Acceleration Conference, Knoxville, TN, USA, August 21-25, 2006.

7) M. Wilson, et. al., "HIF Impulse Injector Design, Construction, and Checkout," UCRL-ID-130764, 1998.

8) Matt Myers of NRL (Elektra Facility) provided the Double Eagle Brand velvet. 